\title{
Ultrahigh-Throughput Production of Monodisperse and Multifunctional Janus Microparticles Using in-Air Microfluidics
}

\author{
Tom Kamperman, ${ }^{*}{ }^{\dagger} \odot$ Vasileios D. Trikalitis, ${ }^{\dagger}$ Marcel Karperien, ${ }^{\dagger}$ Claas Willem Visser, ${ }^{\ddagger}, \S$ \\ and Jeroen Leijten ${ }^{*} \dagger$
}

\begin{abstract}
${ }^{\dagger}$ Department of Developmental BioEngineering, Technical Medical Centre, and ${ }^{\S}$ Physics of Fluids Group, Faculty of Science and Technology, University of Twente, Drienerlolaan 5, 7522 NB Enschede, The Netherlands

${ }^{\ddagger}$ Wyss Institute for Biologically Inspired Engineering and John A. Paulson School of Engineering and Applied Sciences, Harvard University, Cambridge, Massachusetts 02138, United States
\end{abstract}

\section{Supporting Information}

ABSTRACT: Compartmentalized Janus microparticles advance many applications ranging from chemical synthesis to consumer electronics. Although these particles can be accurately manufactured using microfluidic droplet generators, the per-nozzle throughputs are relatively low $(\sim \mu \mathrm{L} / \mathrm{min})$. Here, we use "in-air microfluidics" to combine liquid microjets in midair, thereby enabling orders of magnitude faster production of Janus microparticles $(\sim \mathrm{mL} / \mathrm{min})$ as compared to chip-based microfluidics. Monodisperse Janus microparticles with diameters between 50 and $500 \mu \mathrm{m}$, tunable compartment sizes, and functional cargo are controllably produced. Furthermore, these microparticles are designed as magnetically steerable microreactors, which represents a novel tool to perform enzymatic cascade reactions within continuous fluid flows

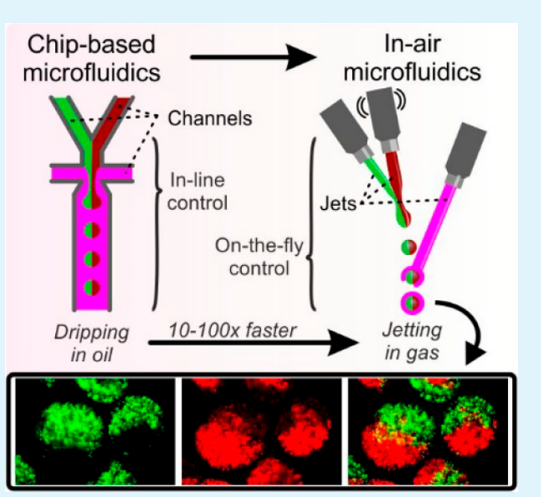

KEYWORDS: liquid jets, compartmentalization, hydrogels, magnetic steering, microreactors

$\mathrm{T}^{\mathrm{k}}$ he anisotropic nature of Janus microparticles offers unique advantages for numerous applications. ${ }^{1}$ Their physical and/or chemical asymmetry has been leveraged to engineer, among others, amphiphilic particulate surfactants for Pickering emulsification, ${ }^{2}$ multicolored voxels for displays, ${ }^{3}$ compartmentalized microreactors as artificial cell mimics, ${ }^{4}$ and steerable microswimmers for targeted cargo delivery. ${ }^{5}$

Traditionally, Janus microparticles are fabricated by immobilizing and partially modifying a particle's surface on an interface in a $2 \mathrm{D}$ manner, which limits the microparticles morphological complexity. ${ }^{6,7}$ More advanced 3D compartmentalized Janus particles can be produced by in-line manipulating, combining, dispersing, and solidifying precursor solutions. This can be achieved using, for example, centrifugation-based dispensing, ${ }^{8}$ electrohydrodynamic cojetting, ${ }^{9}$ and microfluidic droplet generation. ${ }^{10}$ Although microfluidics-based production methods offer the highest control over particle morphology and complexity, ${ }^{11}$ they are limited by relatively low throughputs, which has been challenging their use in largescale applications. ${ }^{12}$ Specifically, to yield a monodisperse product, conventional microfluidic droplet generators are restricted to operating in the dripping regime, which typically limits the per-nozzle microparticle production rates to 1 to 10 $\mu \mathrm{L} / \mathrm{min} .{ }^{13}$ The parallelization of these chips is the main route toward scalable fabrication and parallelization, but this strategy is highly complex and still limited to $<1000$ channels. ${ }^{12,14}$ Although higher microfluidic throughputs can be achieved jetting, this approach is associated with uncontrolled jet breakup causing microparticle polydispersity. ${ }^{13}$ Off-chip jetting methods such as inkjet and vibrating jet technologies, have also been exploited for the rapid formation of monodisperse particles, but these are isotropic (i.e., not Janus) rather than compartmentalized Janus microparticles. ${ }^{15,16}$

In this work, we demonstrate the ultrahigh-throughput onthe-fly fabrication of compartmentalized Janus hydrogel microparticles using "in-air microfluidics" (IAMF). IAMF is based on the controlled collision of at least two liquid microjets in midair, thereby acting as the faster and chip-free counterpart of channel-based microfluidic approaches (Figure S1). ${ }^{17}$ Here, we have exploited this technology for the first time to produce monodisperse Janus microparticles and microfibers by combining three liquid microjets in midair, which allowed for per-nozzle production rates that exceeded conventional chip-based droplet microfluidics by 2 orders of magnitude. To demonstrate the microparticles' functionality, we coencapsulated paramagnetic microparticles and a combination of enzymes in the distinct Janus microcompartments. The resulting particles were leveraged as magnetically steerable microreactors, which represents a novel and effective tool to

Received: March 31, 2018

Accepted: June 28, 2018

Published: June 28, 2018 

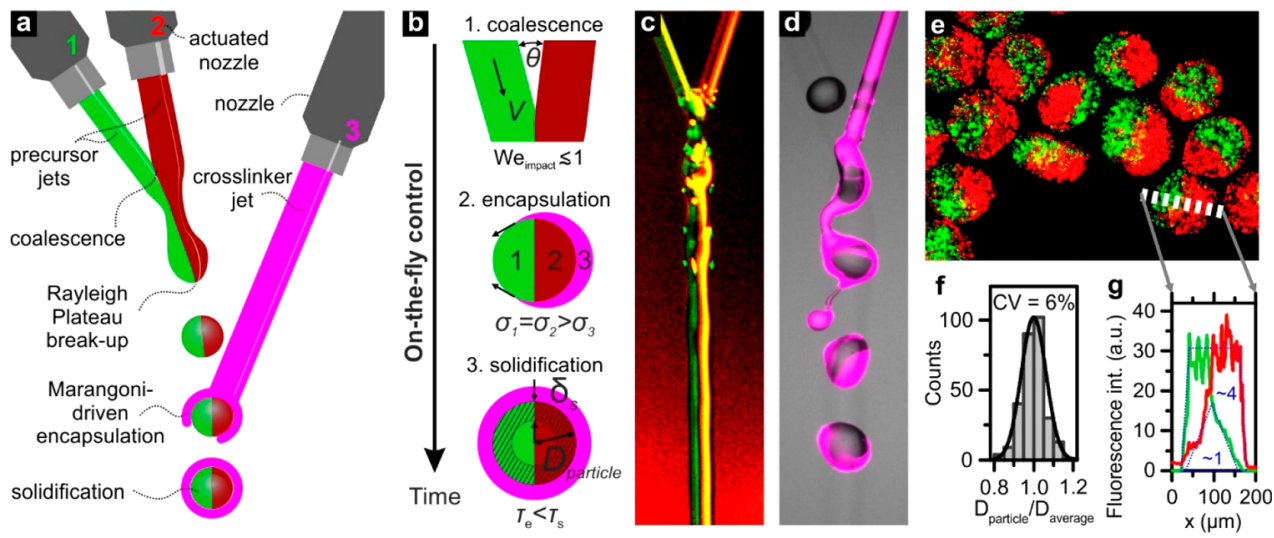

Figure 1. Concept of 3-jet in-air microfluidics (IAMF) for Janus microparticle production. (a) A liquid Janus microjet is formed by coalescing two distinct liquid microjets (green and red) that contain a solid precursor. Piezo-electric microjet actuation generates a train of monodisperse Janus droplets that are subsequently coalesced with a third microjet (magenta) that contains a cross-linker. (b) The key stages of Janus microparticle formation during flight include: (1) Jet-jet and drop-jet coalescence, which requires $W e_{\text {impact }} \lesssim 1$; (2) surface tension gradient-driven encapsulation of the Janus droplets, which requires $\sigma_{1}=\sigma_{2}>\sigma_{3}$; and (3) solidification, such that $\tau_{\text {impact }}<\tau_{\text {sol }}<\tau_{\text {in-air }}$. (c) High-speed microphotograph of two microjets forming a Janus microjet (red and green) with limited initial diffusion. (d) The third microjet (magenta) can encapsulate the in-air formed train of monodisperse droplets, as revealed by high-speed (fluorescence) microscopy. (e) The collected microparticles are characterized by (f) a monodisperse size distribution, as well as (g) a clear compartmentalized morphology. The dotted blue curves indicate that mixed area $\sim 4 \times$ smaller than the total microgel area, which corresponds to a mixed volume of $\sim 1 / 8$ of the microgel's total volume.

perform enzymatic cascade reactions within continuous fluid flows.

To enable the production of compartmentalized Janus particles using IAMF, we pioneered the collision of three liquid microjets in midair (Figure 1a,b and Figure S2). First, a Janus microjet was formed by colliding two precursor solution microjets at a collisional angle $\theta$ smaller than $45^{\circ}$. This resulted in an impact Weber number $W e_{\text {impact }}=\rho D(V \sin \theta)^{2} / \sigma \lesssim 10$, with $\rho, D, V$, and $\sigma$ denoting the liquid microjet's density, diameter, speed, and surface tension, respectively. The modest $W e_{\text {impact }}$ effectively enabled liquid coalescence, while preventing "separation", "splashing", and "bouncing" effects. ${ }^{18,19}$ Highspeed microphotography of colliding colored precursor solutions confirmed the formation of a compound microjet that was composed of two coflowing liquids, herein referred to as the "Janus microjet" (Figure 1c).

By superimposing a periodic wave on a microjet using a piezo-actuated nozzle, a stream of monodisperse droplets can be generated. ${ }^{15}$ Here, we demonstrate that controlled breakup of the Janus microjet is also possible by vibrating only one of the source nozzles, as surface waves continuously travel along the microjet after coalescence with another liquid microjet. Rapid in-air stabilization of the Janus droplets was essential to prevent in-flight and postflight droplet merging. ${ }^{20,21}$ As alginate can be rapidly solidified within milliseconds by cross-linking it with divalent cations, it was selected as the prepolymer solution for the droplets. ${ }^{22}$ To induce on-the-fly solidification, the alginate containing Janus droplets were coalesced with a third liquid microjet (collisional angle $\theta<45^{\circ}$ ) that contained $\mathrm{Ca}^{2+}$ as the cross-linker. To prevent any wasting of liquids during the in-air formation process due to asynchronous droplet streams (i.e., "drop-drop" mode), in-air coalescence events always included at least one intact microjet, herein referred to as "drop-jet" and "jet-jet" mode (Figure S3).

To produce spherical particles, the Janus precursor droplet must be encapsulated (i.e., completely covered) by the crosslinker liquid. ${ }^{17}$ Therefore, the surface tension of the crosslinker microjet was reduced, which induced a Marangoni flow that drove rapid spreading of the $\mathrm{Ca}^{2+}$ solution over the Janus precursor droplet surface. ${ }^{23,24}$ In contrast, the surface tensions of the Janus microjets' polymer precursor solutions were matched to prevent the formation of bowl-shaped or coreshell microparticles. ${ }^{17,25}$ Drop-jet coalescence and encapsulation occur on a time scale $\tau_{\mathrm{e}} \sim\left(\rho \mu D^{4} / \Delta \sigma^{2}\right)^{1 / 3},{ }^{26}$ which typically is a few milliseconds. This is faster than the in-air time of droplets (i.e., $\sim 10$ to $100 \mathrm{~ms}$ ), which grants the on-the-fly encapsulation of the polymer precursor droplets. Indeed, Janus microparticles were successfully produced by sequentially coalescing two $0.5 \%$ alginate containing microjets and a third microjet comprising $0.2 \mathrm{M} \mathrm{CaCl}_{2}$ and $10 \%$ ethanol (Figure 1e). Particle size analysis revealed a monodisperse size distribution, characterized by a coefficient of variation (CV) of $6 \%$ (Figure 1f).

To maintain the microparticles' compartmentalization, it is important that the Janus droplets are solidified before significant mixing occurs. As the alginate precursor solutions are miscible, the two compartments start mixing by diffusion upon first contact over a depth of $\delta_{\mathrm{m}}=\sqrt{D_{\mathrm{m}} t}$, where $D_{\mathrm{m}} \approx 1$ $\times 10^{-9} \mathrm{~m}^{2} \mathrm{~s}^{-1}$ denotes the diffusion of alginate. ${ }^{27}$ Solidification effectively stops the mixing of the alginate compartments and starts upon coalescence with the cross-linker microjet, which is typically $\sim 1 \mathrm{~ms}$ after Janus microjet formation. The solidification rate is limited by the diffusion of $\mathrm{Ca}^{2+}$ into the alginate droplet over a depth of $\delta_{\mathrm{s}}=\sqrt{D_{\mathrm{s}} t}$, where $D_{\mathrm{s}} \approx 1 \times 10^{-9} \mathrm{~m}^{2} \mathrm{~s}^{-1}$ denotes the diffusion constant of $\mathrm{Ca}^{2+}$. ${ }^{22}$ The outer shell of the particle has minimal mass diffusion, because it immediately solidifies. As both diffusion fronts advance at similar rate $\left(D_{\mathrm{m}}\right.$ $\approx D_{\mathrm{s}}$ ), the particle contains a partially mixed core, which is described by a diameter $D_{\text {particle }} / 2$. Therefore, the mixed volume $V_{\mathrm{m}} \sim(D / 2)^{3} \approx 1 / 8$, leaving $\sim 7 / 8$ of the particle pristinely compartmentalized. The majority of the alginate Janus particle's compartmentalization is thus contained, as confirmed using cross-sectional analysis of Janus particles with coencapsulated red and green fluorescent nanoparticles (Figure 1g). 

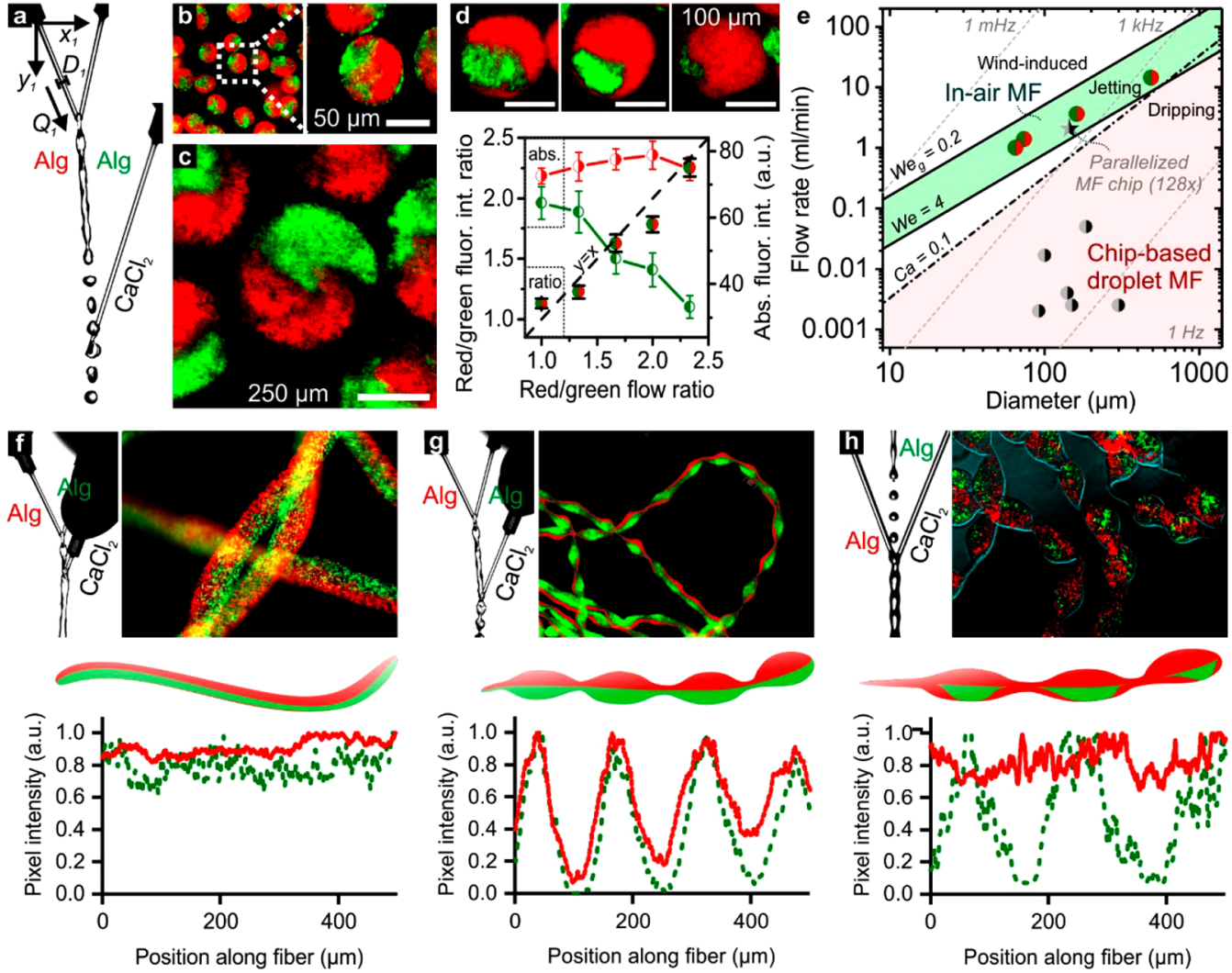

Figure 2. On-the-fly control parameters and throughput. (a) LAMF control parameters include the positions $(x, y)$, diameters $(D)$, and flow rates $(Q)$ of the liquid microjets. The microjet diameters and flow rates could be tuned to change the size of the (b, c) Janus microparticles as well as their (d) individual compartments. (e) Plotting the per-nozzle flow rate as a function of nozzle diameter for in-air and chip-based droplet microfluidics (MF) revealed a significant difference in production throughput. Monodisperse droplet production using chip-based microfluidics relies on dripping. Its production regime is bound by $\mathrm{Ca}=0.1$ and $W e=4$, as indicated by the red shade. In contrast, IAMF is based on jetting, which regime is bound by $W e \gtrsim 4$ and $W e_{\mathrm{g}} \lesssim 0.2$, as indicated by the green shade. The green-red circles are data points obtained using our IAMF setup. The black-gray data points are obtained from previously reported studies on droplet microfluidics for Janus microparticle production (Table S1). The corresponding droplet production frequencies are indicated with gray dashed lines. (f) Positioning the Ca ${ }^{2+}$ containing cross-linker microjet upstream of the alginate containing solid precursor microjets' breakup point resulted in the formation of a Janus microfiber. Alternative positioning resulted in periodically $(\mathrm{g})$ beaded and $(\mathrm{h})$ patched Janus microfibers.

By operating the IAMF setup in "drop-jet" mode (Figure 2a), Janus microparticles with controlled sizes of $74 \pm 6 \mu \mathrm{m}$ $(\mathrm{CV}=7.7 \%), 162 \pm 10 \mu \mathrm{m}(\mathrm{CV}=6.3 \%)$, and $488 \pm 34 \mu \mathrm{m}$ $(\mathrm{CV}=7.0 \%)$, were readily produced by setting the microjets' diameters $D_{1}=D_{2}=50,100$, and $150 \mu \mathrm{m}$, respectively (Figure 2b, c and Figure S4). The relative compartment size of Janus microparticles could also be altered on-the-fly, simply by tuning the flow ratio of the first and second (i.e., precursor) microjet $\left(Q_{1,2}\right)$, which was confirmed by measuring the relative intensities of the distinctly (red and green) fluorescently labeled Janus compartments (Figure 2d).

Plotting the data points from this study together with a representative selection of literature studies, confirmed that IAMF-based production of Janus microparticles is two to 3 orders of magnitude faster as compared to chip-based droplet microfluidics (Figure 2e). IAMF could typically produce tens of milliliters suspension containing more than $50 \%$ (v/v) Janus microparticles using a single setup (i.e., not parallelized) in a few minutes, (Figure S5). This throughput is inherent to jetting into air, for which a liquid Weber number $W e=\rho D V^{2} / \sigma$ $>4$ is required. In contrast, conventional chip-based droplet microfluidics is typically operated in the dripping regime, for which $W e<4$ and Capillary numbers $\mathrm{Ca}=\mu V / \sigma \lesssim 0.1$ are required (but usually $\mathrm{Ca} \ll 0.1$ and $W e \ll 1$ are used), to yield monodisperse microdroplets and microparticles. ${ }^{13,28}$ Importantly, parallelization of microfluidic Janus droplet generators has remained scarce and did not yet reach industrial-scale throughputs. ${ }^{12}$ Notably, a single IAMF setup exceeded the throughput of a previously reported 128 times parallelized chip-based microfluidic Janus droplet generator. The maximum throughput of IAMF-based Janus microparticle production is bound by a gas Weber number $W e_{\mathrm{g}}=\left(\rho_{\mathrm{g}} / \rho_{\mathrm{l}}\right)$ $W e \sim 0.2$, where $\rho_{\mathrm{g}}$ and $\rho_{1}$ are the densities of the liquid jet and the surrounding gas, respectively, above which uncontrolled wind-induced breakup is expected to occur. ${ }^{29}$

In addition to Janus microparticles, various types of microfibers with tunable Janus morphologies could be readily produced by straightforwardly changing the position of the microjets. Positioning the cross-linker microjet upstream of the Janus polymer precursor microjet's breakup point (i.e., "jetjet” mode), a straight Janus microfiber was formed instead of microparticles (Figure 2f). Furthermore, positioning the third microjet only a few droplet diameters before the precursor microjets' breakup point enabled the production of periodically beaded Janus microfibers (Figure $2 \mathrm{~g}$ ). Janus microfibers composed of continuous microfibers containing discrete periodic compartments (i.e., patches) could be produced by positioning the second microjet such that it broke up into a 

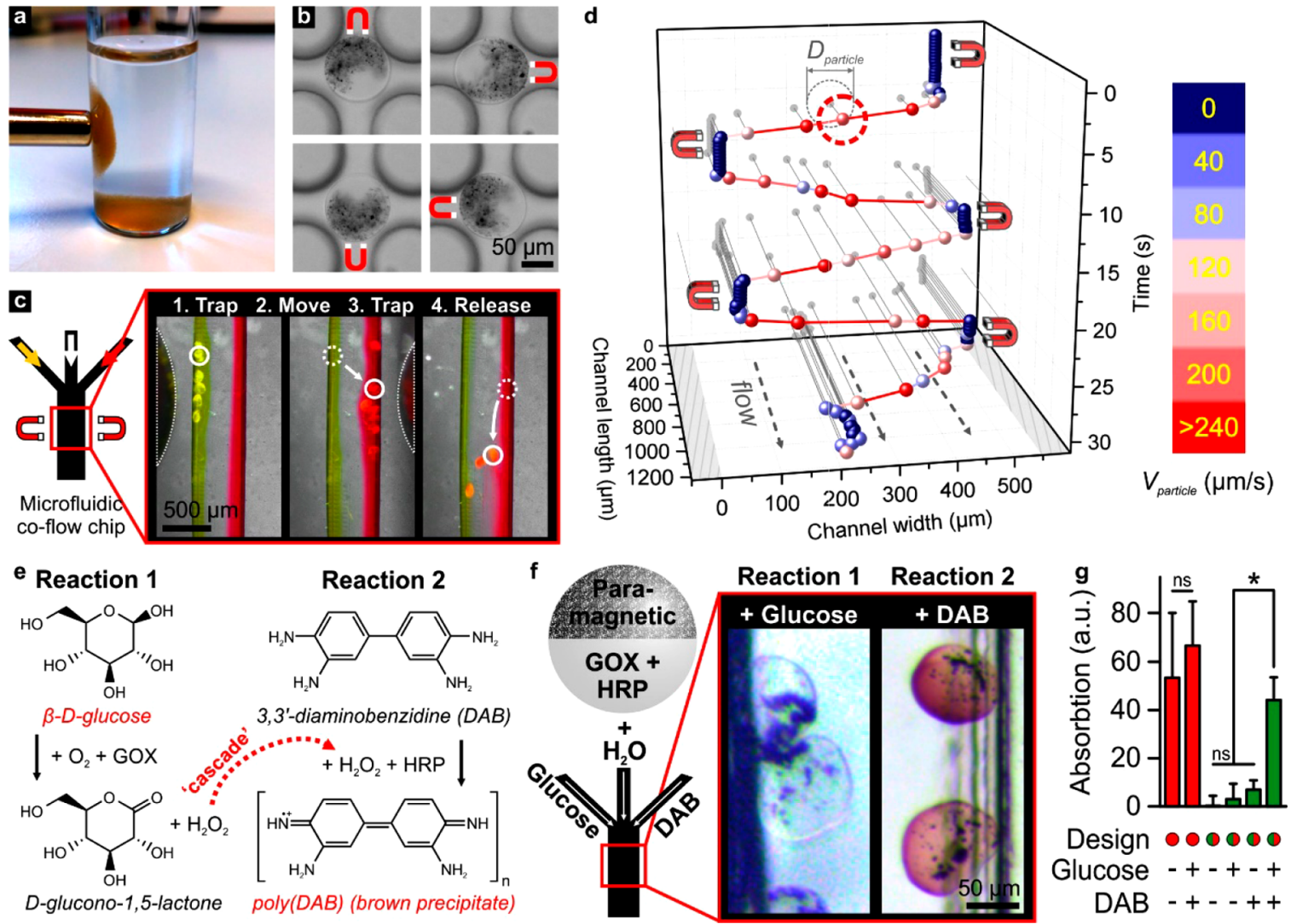

Figure 3. Multifunctional Janus microparticles as magnetically steerable enzymatic microreactors. Using a magnetic field, Janus microparticles with a paramagnetic compartment could be (a) moved and (b) oriented within a static carrier liquid. (c, d) The same particles could also be trapped and moved with micrometer precision within a laminar coflowing liquid. (e) The enzymes glucose oxidase (GOX) and horseradish peroxidase (HRP) can together drive a cascade reaction via the sequential enzymatic production and reaction of $\mathrm{H}_{2} \mathrm{O}_{2}$. In the presence of $\beta$-D-glucose and of 3,3'diaminobenzidine (DAB), a successful cascade reaction is indicated by the formation of poly(DAB), which appears as a brown precipitate. (f) Janus microparticles with paramagnetic and GOX/HRP compartments could be leveraged as steerable microreactors to facilitate a multienzymatic cascade reaction within a laminar coflow of $\beta$-D-glucose, $\mathrm{H}_{2} \mathrm{O}$, and $\mathrm{DAB}$. (g) Compartmentalization of the brown paramagnetic particles and the enzymes was key to enable accurate quantification of the enzymatic cascade reaction product using absorption spectrophotometry. Furthermore, spectrophotometric analysis of various reagent combinations confirmed that both $\beta$-D-glucose and DAB were essential to complete the cascade reaction. "ns" indicates no significance. * indicates significance with $p<0.01$.

droplet train before colliding with the second and third microjet (Figure $2 \mathrm{~h}$ ). Thus, only changing a single parameter (i.e., the interjet position) enabled on-the-fly switching between producing fibrous and particulate matter (Movie S1).

To demonstrate the functionality of the compartmentalized Janus microparticles, we set out to produce steerable microreactors. Particularly, we created microparticles with a physicochemical anisotropy by adding paramagnetic microparticles to one of the solid precursor microjets. The resulting Janus microparticles exhibited paramagnetic properties that allowed for their spatial manipulation and orientation within a static carrier solution in a noninvasive manner via exposure to a magnetic field (Figure 3a, b). We then investigated whether the paramagnetic Janus particles could also be positioned within flowing liquids, which is essential for their application as steerable microreactors within microfluidic devices such as labon-a-chip platforms, as well as large-scale continuous chemical process. The paramagnetic Janus particles were injected into a microfluidic device, which was composed of a channel (500 $\mu \mathrm{m}$ wide, $250 \mu \mathrm{m}$ high) that was connected to three inlets. A laminar coflow of three separated liquids (Reynolds number Re $\sim 1, V \sim 4 \mathrm{~mm} / \mathrm{s}$ ) was generated by pumping aqueous solutions into the inlets. Under flow, the paramagnetic Janus particles could be trapped, moved across fluid flow lines, and released on-demand using a magnetic field $B \sim 0.25 \mathrm{~T}$. This strategy enabled the in situ positioning of the particles within three chemically distinct fluids, as visualized using a laminar triple coflow of yellow, colorless, and red fluids (Figure 3c, Movie S2). Trajectory recordings of the paramagnetic Janus microparticles revealed that they could be trapped indefinitely, but also moved across fluid flow lines with velocities exceeding $240 \mu \mathrm{m} / \mathrm{s}$ (Figure 3d). Magnetic steering could thus be used to switch the Janus microparticles' environment within a single second and without the need for currently employed microfluidic flow control strategies, which are more complex and arduous to operate. Such conventional strategies can be, for example, based on multilayered devices (i.e., Quake valves), electrowetting (i.e., digital microfluidics), or 3D channel surface topographies (i.e., inertial microfluidics). Advantageously, magnetic manipulation is noninvasive and easily automated, which facilitates the integration of this technology into large-scale industrial systems. These features make the steerable Janus particles specifically suitable as microreactors for multistep reactions, also known as cascade reactions, with high spatiotemporal resolution. The ability to tailor individual reaction conditions, as well as the rapid exchange of reactants and reaction products, have primed compartmentalized 
cascade microreactors as powerful tools for, for example, complex sequential enzymatic reactions. ${ }^{30}$

The steerable Janus microparticles were endowed with a chemically functional domain to yield multifunctional particles that could act as steerable multienzymatic microreactors. To achieve this, we discretely coencapsulated paramagnetic microparticles and a combination of two enzymes to enable a multienzymatic cascade reaction, which was accomplished by moving the steerable enzyme-based microreactors across multiple reagent fluid flows. To this end, the enzymes glucose oxidase (GOX) and horseradish peroxidase (HRP) were selected. GOX catalyzes the oxidation of $\beta$-D-glucose to form D-glucono-1,5-lactone and hydrogen peroxide $\left(\mathrm{H}_{2} \mathrm{O}_{2}\right)$, whereas HRP catalyzes the $\mathrm{H}_{2} \mathrm{O}_{2}$-induced oxidation of the chromogenic substrate $3,3^{\prime}$-diaminobenzidine $(\mathrm{DAB})$ to form poly(DAB). Poly $(\mathrm{DAB})$ precipitates and was used to visualize the successful enzymatic cascade reaction based on the colorimetric colorless-to-brown shift (Figure 3e).

The paramagnetic Janus microparticles were positioned inside the channel of a microfluidic device through which a $\beta$ $\mathrm{D}$-glucose solution, water, and a $\mathrm{DAB}$ solution were laminarly coflown. Using a magnetic field, the Janus particles were sequentially trapped within and moved across the reagent flow lines, thereby effectively acting as steerable enzymatic microreactors. A successful enzymatic cascade reaction occurred in the microparticles by sequentially incubating them within the glucose and $\mathrm{DAB}$ containing streams, as revealed by the formation of brown precipitate (Figure $3 \mathrm{f}$ ). Importantly, the compartmentalized Janus design was essential for the accurate quantification of the multienzymatic reaction product. Homogeneous encapsulation of the brown paramagnetic particles hindered the accurate visual readout of the multienzymatic reaction product. In fact, spectrophotometric analysis of the negative control (i.e., without glucose and $\mathrm{DAB}$ ) revealed a high absorption (i.e., false positive) in the presence of paramagnetic microparticles (Figure $3 g$ ). Therefore, spatially distinct encapsulation of the paramagnetic particles and enzymes in the respective Janus compartments was key to enable an accurate visual readout of the colorimetric assay's reaction product. The multienzyme dependency of the reaction was confirmed by incubating the paramagnetic Janus microparticles with $\beta$-D-glucose or DAB alone (Figure $3 \mathrm{~g}$ ). As magnetic manipulation is easily automated and integrated in large-scale applications, our IAMF-produced steerable microreactors represent a promising tool to upscale multienzyme microreactor chemistry.

In conclusion, we pioneered a feasible method for the ultrahigh-throughput production of various Janus microparticles and microfibers, of which the function, size, and compartmentalization can be tailored and spatially organized. By using IAMF-based droplet generation, the per-nozzle Janus particle production rate was increased by two to 3 orders of magnitude as compared to conventional chip-based droplet microfluidic platforms, which is expected to facilitate IAMF's integration into large-scale industrial applications. To demonstrate the functionality of our approach, we produced, for the first time, magnetically steerable multienzymatic microreactors by coencapsulating magnetic microparticles and multiple enzymes into distinct compartments of Janus microparticles. This approach yielded physicochemical anisotropic microparticles that acted as magnetically steerable microreactors for enzymatic cascade reactions. The facile, ultrahigh-throughput, and multifunctional nature of IAMF-based Janus production platform primes this novel technology for its widespread integration in industrial and clinical applications, for example, to facilitate multienzymatic reactions in continuous flow systems.

\section{ASSOCIATED CONTENT}

Supporting Information

The Supporting Information is available free of charge on the ACS Publications website at DOI: 10.1021/acsami.8b05227.

Experimental Section, Figures S1-S5, Table S1 (PDF)

Movie S1 (AVI)

Movie S2 (AVI)

\section{AUTHOR INFORMATION}

\section{Corresponding Authors}

*E-mail: t.kamperman@utwente.nl.

*E-mail: j.c.h.leijten@utwente.nl.

ORCID

Tom Kamperman: 0000-0002-2835-6709

\section{Author Contributions}

Conception by T.K., C.W.V., and J.L. Experimental design by T.K., C.W.V., and J.L. Experiments performed by T.K., V.T., and C.W.V. Data interpretation by all authors. Manuscript written by T.K., C.W.V., and J.L. Supervision and revisions by M.K. and J.L.

\section{Notes}

The authors declare the following competing financial interest(s): T.K., M.K., and C.W.V. are co-inventors on a patent based on this work filed by the University of Twente (WO2017167798). T.K. and C.W.V. are co-founders and directors of the company IamFluidics BV, which is commercializing the IAMF technology. The authors declare no other conflicting interests.

\section{ACKNOWLEDGMENTS}

T.K., M.K., C.W.V., and J.L. acknowledge financial support from the MIRA Institute for Biomedical Technology and Technical Medicine. M.K. acknowledges financial support from the Dutch Arthritis Foundation (\#12-2-411 and LLP-25). J.L. acknowledges financial support from an Innovative Research Incentives Scheme Veni award (\#14328) from The Netherlands Organization for Scientific Research (NWO), the European Research Council (ERC, Starting Grant, \#759425), and the Dutch Arthritis Foundation (\#12-2-411 and \#17-1405).

\section{REFERENCES}

(1) Walther, A.; Muller, A. H. E. Janus Particles: Synthesis, SelfAssembly, Physical Properties, and Applications. Chem. Rev. 2013, 113 (7), 5194-5261.

(2) Park, B. J.; Brugarolas, T.; Lee, D. Janus Particles at an Oil-Water Interface. Soft Matter 2011, 7 (14), 6413-6417.

(3) Komazaki, Y.; Hirama, H.; Torii, T. Electrically and Magnetically Dual-Driven Janus particles for Handwriting-Enabled Electronic Paper. J. Appl. Phys. 2015, 117 (15), 154506.

(4) Schattling, P.; Dreier, C.; Stadler, B. Janus Subcompartmentalized Microreactors. Soft Matter 2015, 11 (26), 5327-5335.

(5) Baraban, L.; Makarov, D.; Streubel, R.; Monch, I.; Grimm, D.; Sanchez, S.; Schmidt, O. G. Catalytic Janus Motors on Microfluidic Chip: Deterministic Motion for Targeted Cargo Delivery. ACS Nano 2012, 6 (4), 3383-3392. 
(6) Loget, G.; Kuhn, A. Bulk Synthesis of Janus Objects and Asymmetric Patchy Particles. J. Mater. Chem. 2012, 22 (31), 1545715474.

(7) Perro, A.; Reculusa, S.; Ravaine, S.; Bourgeat-Lami, E.; Duguet, E. Design and Synthesis of Janus Micro- and Nanoparticles. J. Mater. Chem. 2005, 15 (35-36), 3745-3760.

(8) Maeda, K.; Onoe, H.; Takinoue, M.; Takeuchi, S. Controlled Synthesis of 3D Multi-Compartmental Particles with CentrifugeBased Microdroplet Formation from a Multi-Barrelled Capillary. Adv. Mater. 2012, 24 (10), 1340-1346.

(9) Roh, K. H.; Martin, D. C.; Lahann, J. Biphasic Janus Particles with Nanoscale Anisotropy. Nat. Mater. 2005, 4 (10), 759-763.

(10) Lone, S.; Cheong, I. W. Fabrication of Polymeric Janus Particles by Droplet Microfluidics. RSC Adv. 2014, 4 (26), 1332213333.

(11) Choi, A.; Seo, K. D.; Kim, D. W.; Kim, B. C.; Kim, D. S. Recent Advances in Engineering Microparticles and Their Nascent Utilization in Biomedical Delivery and Diagnostic Applications. Lab Chip 2017, 17 (4), 591-613.

(12) Holtze, C. Large-Scale Droplet Production in Microfluidic Devices-an Industrial Perspective. J. Phys. D: Appl. Phys. 2013, 46 (11), 114008

(13) Nunes, J. K.; Tsai, S. S.; Wan, J.; Stone, H. A. Dripping and Jetting in Microfluidic Multiphase Flows Applied to Particle and Fiber Synthesis. J. Phys. D: Appl. Phys. 2013, 46 (11), 114002.

(14) Nisisako, T. Recent Advances in Microfluidic Production of Janus Droplets and Particles. Curr. Opin. Colloid Interface Sci. 2016, 25, $1-12$.

(15) Whelehan, M.; Marison, I. W. Microencapsulation using Vibrating Technology. J. Microencapsulation 2011, 28 (8), 669-688.

(16) Xu, T.; Kincaid, H.; Atala, A.; Yoo, J. J. High-Throughput Production of Single-Cell Microparticles Using an Inkjet Printing Technology. Journal of Manufacturing Science and Engineering 2008, 130 (2), 021017.

(17) Visser, C. W.; Kamperman, T.; Karbaat, L. P.; Lohse, D.; Karperien, M. In-air Microfluidics Enables Rapid Fabrication of Emulsions, Suspensions, and 3D Modular (Bio)materials. Sci. Adv. 2018, 4 (1), eaao1175.

(18) Wadhwa, N.; Vlachos, P.; Jung, S. Noncoalescence in the Oblique Collision of Fluid Jets. Phys. Rev. Lett. 2013, 110 (12), 124502.

(19) Chen, C.; Chiu, S.-L.; Lin, T.-H. Collisions of a String of Water Drops on a Water Jet of Equal Diameter. Exp. Therm. Fluid Sci. 2006, 31,75 .

(20) Brandenberger, H.; Nussli, D.; Piech, V.; Widmer, F. Monodisperse Particle Production: A Method to Prevent Drop Coalescence using Electrostatic Forces. J. Electrost. 1999, 45 (3), 227-238.

(21) Suverkrup, R.; Eggerstedt, S. N.; Gruner, K.; Kuschel, M.; Sommerfeld, M.; Lamprecht, A. Collisions in Fast Droplet Streams for the Production of Spherolyophilisates. Eur. J. Pharm. Sci. 2013, 49 (4), 535-541.

(22) Lin, S. H. Gelation of Sodium Alginate in a Batch Process. Chem. Eng. Sci. 1991, 46 (2), 651-655.

(23) Blanchette, F. Simulation of Mixing within Drops due to Surface Tension Variations. Phys. Rev. Lett. 2010, 105 (7), 074501.

(24) Planchette, C.; Lorenceau, E.; Brenn, G. Liquid Encapsulation by Binary Collisions of Immiscible Liquid Drops. Colloids Surf., A 2010, 365 (1), 89-94.

(25) Hayakawa, M.; Onoe, H.; Nagai, K. H.; Takinoue, M. Complex-Shaped Three-Dimensional Multi-Compartmental Microparticles Generated by Diffusional and Marangoni Microflows in Centrifugally Discharged Droplets. Sci. Rep. 2016, 6, 20793.

(26) Capelleveen, B. F. v.; Koldeweij, R. B. J.; Lohse, D.; Visser, C. W. On the Universality of Marangoni-Driven Spreading along LiquidLiquid Interfaces. Arxiv:1712.03192 2017.

(27) Ribeiro, A. C. F.; Sobral, A. J. F. N.; Simões, S. M. N.; Barros, M. C. F.; Lobo, V. M. M.; Cabral, A. M. T. D. P. V.; Veiga, F. J. B.; Santos, C. I. A. V.; Esteso, M. A. Transport Properties of Aqueous
Solutions of Sodium Alginate at 298.15K. Food Chem. 2011, 125 (4), $1213-1218$

(28) Yan, Z.; Clark, I. C.; Abate, A. R. Rapid Encapsulation of Cell and Polymer Solutions with Bubble-Triggered Droplet Generation. Macromol. Chem. Phys. 2017, 218 (2), 1600297.

(29) van Hoeve, W.; Gekle, S.; Snoeijer, J. H.; Versluis, M.; Brenner, M. P.; Lohse, D. Breakup of Diminutive Rayleigh Jets. Phys. Fluids 2010, 22 (12), 122003.

(30) Gruber, P.; Marques, M. P. C.; O’Sullivan, B.; Baganz, F.; Wohlgemuth, R.; Szita, N. Conscious Coupling: The Challenges and Opportunities of Cascading Enzymatic Microreactors. Biotechnol. J. 2017, 12 (7), 1700030. 